\title{
Deformations of Super Riemann Surfaces
}

\author{
Holger Ninnemann \\ II. Institut für Theoretische Physik, Universität Hamburg, Luruper Chaussee 149, 2000 Hamburg \\ 50 , Germany
}

Received March 7, 1991; in revised form June 5, 1992

\begin{abstract}
Two different approaches to (Kostant-Leites-) super Riemann surfaces are investigated. In the local approach, i.e. glueing open superdomains by superconformal transition functions, deformations of the superconformal structure are discussed. On the other hand, the representation of compact super Riemann surfaces of genus greater than one as a fundamental domain in the Poincare upper half-plane provides a simple description of super Laplace operators acting on automorphic $p$-forms.

Considering purely odd deformations of super Riemann surfaces, the number of linear independent holomorphic sections of arbitrary holomorphic line bundles will be shown to be independent of the odd moduli, leading to a simple proof of the Riemann-Roch theorem for compact super Riemann surfaces. As a further consequence, the explicit connections between determinants of super Laplacians and Selberg's super zeta functions can be determined, allowing to calculate at least the 2-loop contribution to the fermionic string partition function.
\end{abstract}

\section{Introduction}

In recent years, the theory of super Riemann surfaces has gained some attention, mainly motivated by the study of fermionic strings and superconformal field theories. As is well known, Polyakov's functional integral describing the $g$-loop contribution in the perturbation expansion of the fermionic string partition function can be reduced to a finite dimensional integral over super moduli space $\mathscr{M}_{g}$, the space parametrizing all super Riemann surfaces of genus $g$. The integrand contains some determinants of super Laplace operators acting on $p$-forms, which may be expressed via Selberg's super zeta functions in the case of genus greater than one. The explicit structure of these relations depends on the number of linear independent zero modes of the super Laplacian.

Two basically different approaches to supermanifolds exist: the one introduced by DeWitt [8] and the theory of graded manifolds in the sense of Kostant and Leites $[27,28]$. I will follow the second approach, because it allows to use a lot of 
standard methods of complex analysis and algebraic topology, generalized to the $\mathbb{Z}_{2}$-graded case. Furthermore the Kostant-Leites description seems to be physically more natural (see e.g. [30]).

Besides having physical applications, the theory of super Riemann surfaces is interesting on its own from the mathematical viewpoint. The intention of this paper is, on the one hand, to present two different descriptions of (Kostant-Leites-) super Riemann surfaces and their connections: namely the representation as a collection of open superdomains glued via superconformal transition functions, and for genus greater than one, using uniformization, the representation as a fundamental domain in the Poincaré upper half-plane. On the other hand, considering purely odd deformations of a super Riemann surface, it will be shown that the number of linearly independent holomorphic sections of arbitrary holomorphic line bundles is independent of the odd moduli, a statement allowing to generalize the RiemannRoch theorem to the case of compact super Riemann surfaces [35].

This paper is organized as follows. At first, I review some basic facts concerning supermanifolds and Berezin integration. After defining super Riemann surfaces in the local approach and holomorphic line bundles over it, deformations of super Riemann surfaces respecting the superconformal structure will be discussed in order to determine the dimension of super moduli space. The following section deals with hyperbolic geometry on the super Poincaré upper half-plane and automorphic forms. Finally the connection to fermionic string theory will be pointed out, ending with the calculation of the 2-loop contribution to the fermionic partition function.

\section{Supermanifolds}

In this section some basic facts about supermanifolds will be reviewed, e.g. splitness, Batchelor's theorem, volume forms and Berezin integration. A more detailed treatment can be found in Leites [27] and Manin [28].

At first recall the following

Definition. $A \mathbb{Z}_{2}$-graded commutative ringed space $\left(M, \mathscr{S}_{M}\right)$, i.e. a topological manifold $M$ and a sheaf $\mathscr{S}_{M}$ of supercommutative rings, is called a supermanifold of dimension $m \mid n$, if

(i) $\left(M, \mathscr{S}_{M} / \mathscr{N}\right)$ is an ordinary $\mathscr{S}_{M} / \mathscr{N}$-manifold of dimension $m . \mathscr{N} \subset \mathscr{S}_{M}$ denotes the subsheaf of nilpotents $\mathscr{N}=\mathscr{S}_{M, \overline{1}} \oplus\left(\mathscr{S}_{M, \overline{1}}\right)^{2}$.

(ii) $\mathscr{N} / \mathscr{N}^{2}$ is a locally free sheaf of $\mathscr{S}_{M} / \mathscr{N}$-moduls of rang $0 \mid n$.

(iii) The structure sheaf $\mathscr{S}_{M}$ is locally decomposable, i.e. locally isomorphic to the symmetric algebra of $\mathcal{N} / \mathscr{N}^{2}$,

$$
\left.\mathscr{S}_{M \mid U} \cong S_{\mathscr{S}_{M} / \mathscr{N}}\left(\mathcal{N} / \mathscr{N}^{2}\right)\right|_{U}, \quad U \subset M .
$$

The ordinary manifold $\left(M, \mathscr{S}_{M} / \mathscr{N}\right)$ is often called the reduced manifold $M_{\text {red }}$ and arises (loosely speaking) by "setting all nilpotents of $\mathscr{S}_{M}$ to zero." Condition (iii) means that the structure sheaf $\mathscr{S}_{M}$ is locally generated by linear independent sections $\left(x^{1}, \ldots, x^{m}, \xi^{1}, \ldots, \xi^{n}\right)$ which are subject to no other relations than supercommutativity. Therefore locally every superfunction (i.e. section of $\mathscr{S}_{M}$ ) can be uniquely represented as

$$
f(x, \xi)=f_{0}(x)+\xi^{i} f_{i}(x)+\xi^{i} \xi^{j} f_{i j}(x)+\cdots, \quad f_{0}, f_{i}, f_{i j} \in \Gamma\left(U, \mathscr{S}_{M, \text { 'red }}\right) .
$$


A supermanifold is said to be decomposable or split, if the isomorphism (2.1) holds globally. The meaning of this notion can be easily seen by considering the effect on the transition functions between two intersecting open domains $U, V \subset M$. Let $\left(x^{1}, \ldots, x^{m}, \xi^{1}, \ldots, \xi^{n}\right)$ and $\left(y^{1}, \ldots, y^{m}, \eta^{1}, \ldots, \eta^{n}\right)$ be local coordinates on $U$ and $V$, respectively. On a general supermanifold they are related by

$$
\begin{aligned}
y^{k}(x, \xi) & =a_{0}^{k}(x)+\xi^{i} \xi^{j} a_{i j}^{k}(x)+\cdots, \\
\eta^{l}(x, \xi) & =\xi^{i} b_{i}^{l}(x)+\xi^{i} \xi^{j} \xi^{k} b_{i j k}^{l}(x)+\cdots .
\end{aligned}
$$

However on a split supermanifold the global $\mathbb{Z}$-grading of $\mathscr{S}_{M}$ results in a reduction of $(2.3)$ to

$$
y^{k}(x, \xi)=a_{0}^{k}(x), \quad \eta^{k}(x, \xi)=\xi^{i} b_{i}^{l}(x) .
$$

For this reason on a split supermanifold the component decomposition (2.2) of an arbitrary superfunction $f \in \Gamma\left(M, \mathscr{S}_{M}\right)$ is globally well defined, and each component is a section of a vector bundle on the reduced manifold $M_{\text {red }}$. Whereas complex analytic supermanifolds are in general not decomposable, in the differentiable category one has the following

Theorem. (Batchelor [5]). Every differentiable supermanifold $\left(M, \mathscr{E}_{M}\right)$ is decomposable.

For defining integration on supermanifolds, remember that objects which can be integrated over an ordinary (orientable, compact) manifold $M_{\text {red }}$ are sections of the maximum exterior power of the cotangent bundle

locally

$$
\sigma \in \Gamma\left(M_{\mathrm{red}}, \Lambda^{\max } \Omega^{1} M_{\mathrm{red}}\right)
$$

$$
\sigma=f(x) d x^{1} \wedge \ldots \wedge d x^{m} .
$$

Therefore under a change of coordinates $f(x)$ is multiplied by the Jacobian.

On a supermanifold, however, because of the presence of anticommuting coordinates, no maximum exterior power of the cotangent bundle exists, but volume forms can be consistently defined to look locally like

$$
\sigma=D^{*}(d x, d \xi) \cdot f(x, \xi)
$$

with $f$ transforming on the intersection of two coordinate neighbourhoods according to

$$
f(y, \eta)=\left\{\operatorname{sdet} \frac{\partial(y, \eta)}{\partial(x, \xi)}\right\}^{-1} f(x, \xi) .
$$

The sheaf of volume forms is also denoted as the Berezinian Ber $M$ of $M$

$$
\sigma \in \Gamma(M, \operatorname{Ber} M) \text {. }
$$

Finally, the Berezin integral over a (super-) domain $\left(U, \mathscr{S}_{M \mid U}\right)$ with local coordinates $\left(x^{1}, \ldots, x^{m}, \xi^{1}, \ldots, \xi^{n}\right)$ can be defined as [16]

$$
\int_{\left(U, \mathscr{S}_{M \mid U}\right)} \sigma:=\int_{U} d x^{1} \wedge \ldots \wedge d x^{m} \frac{\partial^{n}}{\partial \xi^{1} \ldots \partial \xi^{n}} f(x, \xi) .
$$


This definition is independent of the choice of local coordinates, and obviously the Berezin integral depends only on the coefficient function of the maximum degree monomial $\xi^{1} \ldots \xi^{n}$ in the component decomposition of $f(x, \xi)$. In complete analogy to the classical case, the integral over the whole supermanifold $\left(M, \mathscr{S}_{M}\right)$ is obtained by glueing superdomains using a partition of unity and the additivity of the Berezin integral.

\section{Super Riemann Surfaces}

Imposing a superconformal structure on a complex 1|1-dimensional supermanifold leads to an object, which in spite of having a complex dimension greater than one, shows many features of ordinary Riemann surfaces. This construction enables one to build a first order differential operator valued in the half-volume forms, a fact being of some interest in fermionic string theory $[16,17,18]$. From the viewpoint of string theory, the presence of the gravitino field makes it necessary to have additional odd parameters besides the odd coordinates. It is therefore quite natural to consider families of super Riemann surfaces. Finally in this section some basic properties of holomorphic line bundles over families of super Riemann surfaces will be treated.

\subsection{Superconformal Structure. More precisely one has the following [15].}

Definition. A super Riemann surface $\left(M, \mathcal{O}_{M}\right)$ is a complex 1|1-dimensional supermanifold, with the property that in local coordinates

(i) the transition functions are holomorphic

$$
z^{\prime}=f(z, \theta), \quad \theta^{\prime}=\psi(z, \theta),
$$

(supercomplex structure),

(ii) the differential operator $D=\partial_{\theta}+\theta \partial_{z}$ transforms homogeneously

$$
D^{\prime} \propto D,
$$

(superconformal structure).

Since $D^{2}=\partial_{z}$ this means: there exists on a super Riemann surface a nonintegrable distribution of rank $0 \mid 1$, and therefore no single "good" coordinate $\eta$ can be found to write $D$ as $D=\partial_{\eta}[18]$.

Using the chain rule one finds

$$
D=\left(D \theta^{\prime}\right) D^{\prime}+\left(D z^{\prime}-\theta^{\prime} D \theta^{\prime}\right) D^{\prime 2},
$$

so the condition (3.2) characterizing superconformal coordinate transformations can also be written in the form

$$
D z^{\prime}=\theta^{\prime} D \theta^{\prime} .
$$

Calculating the Jacobian of the coordinate transformations

$$
\operatorname{sdet} \frac{\partial\left(z^{\prime}, \theta^{\prime}\right)}{\partial(z, \theta)}=D \theta^{\prime},
$$


the transformation law for the $D$-operator reads explicitly

$$
D^{\prime}=\left\{\operatorname{sdet} \frac{\partial\left(z^{\prime}, \theta^{\prime}\right)}{\partial(z, \theta)}\right\}^{-1} D .
$$

With regard to (2.7) this relation shows that

$$
\hat{\partial}:=D^{*}(d z, d \theta) \otimes D
$$

is an invariantly defined first order differential operator, valued in the "half-volume forms" [18]. Such a differential operator can be considered as an analogue of the Cauchy-Riemann operator on ordinary Riemann surfaces, because for an arbitrary superfunction $f$,

$$
D^{*}(d z, d \theta) D^{*}(d \bar{z}, d \bar{\theta}) \cdot(D f)(\bar{D} f)
$$

is a volume form, which can be integrated over a super Riemann surface using no extra information besides the supercomplex and superconformal structure and containing only first order derivatives. This is a special feature of super Riemann surfaces. On ordinary complex two-dimensional manifolds no first order differential operator valued in the half-volume forms exists.

The possibility to define a functional on the set of functions, which is independent of the local structure of the super Riemann surface and is invariant under worldsheet supersymmetry transformations, is the reason for considering super Riemann surfaces in the context of fermionic string theory.

3.2. Families and Line Bundles. So far every super Riemann surface, defined as described in the previous section, is trivially split, for if the odd dimension equals one, the coordinate transformations (3.1) are by reasons of parity necessarily of the form

$$
z^{\prime}=f_{z}(z), \quad \theta^{\prime}=\theta f_{\theta}(z)
$$

But for applications in the fermionic string theory there is the need for allowing the coefficient functions of a superfunction (2.2) to have odd parity. This demand can only be realized by introducing families of super Riemann surfaces.

Definition. Let $\varpi$ be a submersive map between complex superspaces

$$
(X, \mathscr{A}) \stackrel{\varpi=(\pi, \psi)}{\longrightarrow}(Y, \mathscr{B})
$$

such that $\pi$ is proper. Furthermore let

$$
\operatorname{dim}(X, \mathscr{A})-\operatorname{dim}(Y, \mathscr{B})=1 \mid 1 .
$$

The map $\varpi$ is called a family of compact super Riemann surfaces, if there exists a $0 \mid 1$-dimensional distribution $\mathscr{D}$ in the relative tangent sheaf $\mathscr{T}_{\text {rel }} X$, such that the supercommutator $\bmod \mathscr{D}$

$$
[,]_{\mathscr{D}}: \mathscr{D} \otimes_{\mathscr{A}} \mathscr{D} \rightarrow \mathscr{T}_{\text {rel }} X / \mathscr{D}
$$

is an isomorphism. Here the relative tangent sheaf is defined as the sheaf $\mathscr{T}_{\text {rel }} X \subset \mathscr{T} X$ of derivations which annihilate $\psi(\mathscr{B})$. Finally call sections $z, \theta$ of $\mathscr{A}$, such that $d z, d \theta$ are a basis for $\mathscr{T}_{\mathrm{rel}}^{*} X$, a relative coordinate system $[13,26]$. 
The connection with the previous definition is given by a lemma of LeBrun and Rothstein [26], which states that for every family of compact super Riemann surfaces a relative coordinate system $(z, \theta)$ can be found, such that $\mathscr{D}$ is generated by $\partial_{\theta}+\theta \partial_{z}$. In this sense a super Riemann surface, defined as in the beginning of Sect. 3.1, agrees with a family of super Riemann surfaces, where $(Y, \mathscr{B})$ consists of a single point, and thus will be further on denoted as a "single" or "isolated" super Riemann surface.

If only local properties of the parameter space $(Y, \mathscr{B})$ are of interest, it is possible [34] to consider single super Riemann surfaces with enlarged structure sheaf instead of families of super Riemann surfaces, i.e. demanding a local isomorphism

$$
\left.\mathcal{O}_{M \mid U} \cong S_{\mathcal{O}_{M} / \mathcal{N}}\left(\mathscr{N} / \mathscr{N}^{2}\right)\right|_{U} \otimes \Lambda_{\mathcal{O}_{M} / \mathcal{N}}\left(\mathbb{R}^{n}\right), \quad U \subset M,
$$

which provides the structure sheaf with the necessary additional odd parameters.

The explicit form of superconformal coordinate transformations can be derived by imposing the condition (3.4) on holomorphic transition functions

$$
z^{\prime}=f(z)+\theta \psi(z) \sqrt{\frac{\partial f(z)}{\partial z}}, \quad \theta^{\prime}=\psi(z)+\theta \sqrt{\frac{\partial f(z)}{\partial z}+\psi(z) \frac{\partial \psi(z)}{\partial z}} .
$$

Here the convention is used that functions with values of odd parity are denoted by Greek letters.

Let $\varpi: X \rightarrow Y$ be a differentiable family of compact super Riemann surfaces, i.e. the map $\varpi$ is differentiable. A holomorphic line bundle $L$ over $X$ is completely fixed by specifying a 1-cocycle $\left\{g_{\alpha \beta}\right\}$ with coefficients in $\mathcal{O}^{\times}$, the sheaf of even holomorphic functions $f$ such that $f(p) \bmod \mathscr{N} \neq 0, \forall p \in X$. Defining an equivalence relation

$$
\begin{gathered}
\left(x_{\alpha}, \sigma_{\alpha}\right) \sim\left(x_{\beta}, \sigma_{\beta}\right): \Leftrightarrow\left(x_{\alpha}, \sigma_{\alpha}\right)=\left(x_{\beta}, g_{\alpha \beta} \sigma_{\beta}\right), \\
\forall\left(x_{\alpha}, \sigma_{\alpha}\right),\left(x_{\beta}, \sigma_{\beta}\right) \in\left(\mathscr{U}_{\alpha} \cap \mathscr{U}_{\beta}\right) \times \mathbb{C}^{1 \mid 1}
\end{gathered}
$$

for $\left(\mathscr{U}_{\alpha}\right)_{\alpha \in I}$ an open covering of $X$, the bundle $L$ can be represented as the quotient space

$$
L=\biguplus_{\alpha \in I} \mathscr{U}_{\alpha} \times \mathbb{C}^{1 \mid 1 /} \sim
$$

In the further text I will use a notation which discriminates more explicitly between the relative fiber coordinates $z, \theta$ and the coordinates $t^{i}, \zeta^{j}$ of the parameter space $Y$ of the family $\varpi$. Remember that $\varpi$ is a submersive differentiable map and is therefore locally a projection. For a sufficiently small open set $\Delta \subset Y$ it is possible [25] to consider each fiber $M_{t, \zeta}:=\varpi^{-1}\left(t^{i}, \zeta^{j}\right), \forall\left(t^{i}, \zeta^{j}\right) \in \Delta$ as a union

$$
M_{t, \zeta}=\bigcup_{\alpha \in I} U_{\alpha}, \quad\left(t^{i}, \zeta^{j}\right) \in \Delta,
$$

where each $U_{\alpha}$ is independent of the parameters $\left(t^{i}, \zeta^{j}\right)$, only the way of glueing the $\left(U_{\alpha}\right)_{\alpha \in I}$ by superconformal transition functions of the form (3.14) depends on the parameters $\left(t^{i}, \zeta^{j}\right)$.

The transition functions $g_{\alpha \beta}\left(z_{\beta}, \theta_{\beta} ; t^{i}, \zeta^{j}\right)$ of a holomorphic line bundle $L$ are holomorphic in the relative fiber coordinates $z, \theta$ and differentiable in the moduli 
$t^{i}, \zeta^{j}$. For 'fixed $t^{i}, \zeta^{j}$ the 1-cocycle $\left\{g_{\alpha \beta}\left(z_{\beta}, \theta_{\beta} ; t^{i}, \zeta^{j}\right)\right\}$ can be considered as the defining data of a holomorphic line bundle on the single super Riemann surface $M_{t, \zeta}$ with enlarged structure sheaf.

Rejecting the nilpotent part of the transition functions $\left\{g_{\alpha \beta} \bmod \mathcal{N}\right\}$, a holomorphic line bundle $L$ on $M$ always determines a reduced bundle $L_{\text {red }}$ on $M_{\text {red }}$.

As in the classical case, the canonical bundle $\omega$ of a super Riemann surface $M$ can be defined by splitting the bundle of volume forms ${ }^{1}$ Ber $M$ using the supercomplex structure

$$
\operatorname{Ber} M=: \omega \otimes \bar{\omega} .
$$

Observing that the superdeterminant is a multiplicative homomorphism, relation (2.8) shows the transition functions of the canonical bundle $\omega$ to be

$$
g_{\alpha \beta}=\left\{\operatorname{sdet} \frac{\partial\left(z_{\alpha}, \theta_{\alpha}\right)}{\partial\left(z_{\beta}, \theta_{\beta}\right)}\right\}^{-1} .
$$

I close this section by stating some facts about the Chern class $c(L)$ of a holomorphic line bundle $L$. It is defined as the coboundary map resulting from the exponential sequence on super Riemann surfaces

$$
c: H^{1}\left(M, \mathcal{O}^{\times}\right) \rightarrow H^{2}(M, \mathbb{Z}), \quad L \mapsto \delta^{*}(L) .
$$

Since $\mathbb{Z}$ (like every constant sheaf) is completely determined by the topological structure of $M$, the cohomology group $H^{2}(M, \mathbb{Z})$ can be identified with $H^{2}\left(M_{\text {red }}, \mathbb{Z}\right)$, and one finds [33] that the Chern class of $L$ coincides with the Chern class of the reduced bundle

$$
c(L)=c\left(L_{\mathrm{red}}\right)
$$

Noting that

$$
\operatorname{sdet} \frac{\partial\left(z_{\alpha}, \theta_{\alpha}\right)}{\partial\left(z_{\beta}, \theta_{\beta}\right)} \bmod \mathscr{N}=D_{\beta} \theta_{\alpha} \bmod \mathscr{N}=\sqrt{\frac{\partial f_{\alpha \beta}\left(z_{\beta}\right)}{\partial z_{\beta}}} \bmod \mathscr{N},
$$

the reduced canonical bundle on a super Riemann surface is a spinor bundle on $M_{\text {red }}$

$$
\omega_{\text {red }} \cong K^{\frac{1}{2}},
$$

and therefore Eq. (3.21) yields

$$
c(\omega)=g-1
$$

\section{Deformation of Superconformal Structures}

Deforming the coordinate transformations of a split super Riemann surface in a way which respects the superconformal structure, makes it possible to calculate the dimension of the super moduli space $\mathscr{M}_{g}$, the space parametrizing all super Riemann surfaces of genus $g$. The consideration of purely odd deformations leads to the fact that the number of linear independent holomorphic sections of a line

\footnotetext{
${ }^{1}$ Vector bundles and locally free sheafs are considered to be equivalent in the obvious way.
} 
bundle $L_{t, \zeta}$ over $M_{t, \zeta}$ is independent of the odd moduli $\zeta^{j}$, a statement which can be used to establish a generalized version of the Riemann-Roch theorem, valid on super Riemann surfaces.

4.1. The Dimension of Super Moduli Space. Denoting the derivative with respect to the even coordinate $\partial_{z} f(z)$ by $f^{\prime}(z)$, the clutching functions of a split super Riemann surface $M_{0}$ read

$$
z_{\alpha}=f_{\alpha \beta}\left(z_{\beta}\right), \quad \theta_{\alpha}=\theta_{\beta} \sqrt{f_{\alpha \beta}^{\prime}\left(z_{\beta}\right)} .
$$

They don't contain any additional odd parameters $\zeta^{j}$ besides the odd coordinate $\theta$, so the set of all split super Riemann surfaces is completely described by the reduced super moduli space $\mathscr{M}_{g \text {,red }}$. Furthermore, as can be seen from (4.1), a split super Riemann surface contains exactly the same information as an ordinary Riemann surface equipped with a spin structure to fix the sign ambiguity of the square root.

To find the number of linearly independent parameters describing different superconformal structures, one starts by deforming the transition functions (4.1) up to first order in the parameters $t, \zeta$ [12]. Proceeding this way, it is important to preserve the general form of superconformal clutching functions (3.14)

$$
\begin{aligned}
& z_{\alpha}=f_{\alpha \beta}\left(z_{\beta}\right)+t b_{\alpha \beta}\left(z_{\beta}\right)+\theta_{\beta} \zeta c_{\alpha \beta}\left(z_{\beta}\right) \sqrt{f_{\alpha \beta}^{\prime}\left(z_{\beta}\right)+t b_{\alpha \beta}^{\prime}\left(z_{\beta}\right)}, \\
& \theta_{\alpha}=\theta_{\beta} \sqrt{f_{\alpha \beta}^{\prime}\left(z_{\beta}\right)+t b_{\alpha \beta}^{\prime}\left(z_{\beta}\right)}+\zeta c_{\alpha \beta}\left(z_{\beta}\right) .
\end{aligned}
$$

Considering the cocycle relations on a triple intersection $U_{\alpha} \cap U_{\beta} \cap U_{\gamma}$

$$
\begin{aligned}
& z_{\alpha}\left\{z_{\beta}\left(z_{\gamma}, \theta_{\gamma}\right), \theta_{\beta}\left(z_{\gamma}, \theta_{\gamma}\right)\right\}=z_{\alpha}\left(z_{\gamma}, \theta_{\gamma}\right), \\
& \theta_{\alpha}\left\{z_{\beta}\left(z_{\gamma}, \theta_{\gamma}\right), \theta_{\beta}\left(z_{\gamma}, \theta_{\gamma}\right)\right\}=\theta_{\alpha}\left(z_{\gamma}, \theta_{\gamma}\right)
\end{aligned}
$$

and neglecting terms of second order in $t, \zeta$, leads by comparing coefficients to three independent equations

$$
\begin{aligned}
f_{\alpha \beta}\left\{f_{\beta \gamma}\left(z_{\gamma}\right)\right\} & =f_{\alpha \gamma}\left(z_{\gamma}\right), \\
b_{\alpha \beta}\left\{f_{\beta \gamma}\left(z_{\gamma}\right)\right\}+f_{\alpha \beta}^{\prime}\left\{f_{\beta \gamma}\left(z_{\gamma}\right)\right\} & b_{\beta \gamma}\left(z_{\gamma}\right)=b_{\alpha \gamma}\left(z_{\gamma}\right), \\
c_{\alpha \beta}\left\{f_{\beta \gamma}\left(z_{\gamma}\right)\right\}+\sqrt{f_{\alpha \beta}^{\prime}\left\{f_{\beta \gamma}\left(z_{\gamma}\right)\right\}} c_{\beta \gamma}\left(z_{\gamma}\right) & =c_{\alpha \gamma}\left(z_{\gamma}\right) .
\end{aligned}
$$

The first relation is the usual cocycle condition for the glueing functions $f_{\alpha \beta}$ on the reduced manifold $M_{\text {red }}$, whereas the remaining two equations can also be written as (suppressing the argument $z_{\gamma}$ )

$$
\begin{gathered}
b_{\alpha \beta} \frac{\partial}{\partial z_{\alpha}}+b_{\beta \gamma} \frac{\partial}{\partial z_{\beta}}=b_{\alpha \gamma} \frac{\partial}{\partial z_{\alpha}}, \\
c_{\alpha \beta} \theta_{\alpha} \frac{\partial}{\partial z_{\alpha}}+c_{\beta \gamma} \theta_{\beta} \frac{\partial}{\partial z_{\beta}}=c_{\alpha \gamma} \theta_{\alpha} \frac{\partial}{\partial z_{\alpha}},
\end{gathered}
$$

which show, together with the transformation properties (4.1) of $\theta$, that deformations of the superconformal structure are described by the cocycles

$$
\begin{gathered}
\left\{b_{\alpha \beta} \frac{\partial}{\partial z_{\alpha}}\right\} \in Z^{1}\left(\left(U_{\alpha}\right)_{\alpha \in I}, \mathcal{O}\left(K^{-1}\right)\right), \\
\left\{c_{\alpha \beta} \theta_{\alpha} \frac{\partial}{\partial z_{\alpha}}\right\} \in Z^{1}\left(\left(U_{\alpha}\right)_{\alpha \in I}, \mathcal{O}\left(K^{-\frac{1}{2}}\right)\right) .
\end{gathered}
$$


Under lócal superconformal reparametrizations

$$
\begin{aligned}
& z_{\alpha} \mapsto z_{\alpha}+t s_{\alpha}\left(z_{\alpha}\right)+\theta_{\alpha} \zeta r_{\alpha}\left(z_{\alpha}\right) \sqrt{1+t s_{\alpha}^{\prime}\left(z_{\alpha}\right)}, \\
& \theta_{\alpha} \mapsto \theta_{\alpha} \sqrt{1+t s_{\alpha}^{\prime}\left(z_{\alpha}\right)}+\zeta r_{\alpha}\left(z_{\alpha}\right),
\end{aligned}
$$

these cocycles can be seen to change by coboundaries

$$
\begin{aligned}
\left\{b_{\alpha \beta} \frac{\partial}{\partial z_{\alpha}}\right\} & \mapsto\left\{b_{\alpha \beta} \frac{\partial}{\partial z_{\alpha}}+s_{\alpha} \frac{\partial}{\partial z_{\alpha}}-s_{\beta} \frac{\partial}{\partial z_{\beta}}\right\}, \\
\left\{c_{\alpha \beta} \theta_{\alpha} \frac{\partial}{\partial z_{\alpha}}\right\} & \mapsto\left\{c_{\alpha \beta} \theta_{\alpha} \frac{\partial}{\partial z_{\alpha}}+r_{\alpha} \theta_{\alpha} \frac{\partial}{\partial z_{\alpha}}-r_{\beta} \theta_{\beta} \frac{\partial}{\partial z_{\beta}}\right\},
\end{aligned}
$$

so in fact they define cohomology classes

$$
\begin{gathered}
{\left[b_{\alpha \beta} \frac{\partial}{\partial z_{\alpha}}\right] \in H^{1}\left(M_{\mathrm{red}}, \mathcal{O}\left(K^{-1}\right)\right),} \\
{\left[c_{\alpha \beta} \theta_{\alpha} \frac{\partial}{\partial z_{\alpha}}\right] \in H^{1}\left(M_{\mathrm{red}}, \mathcal{O}\left(K^{-\frac{1}{2}}\right)\right) .}
\end{gathered}
$$

Using the Riemann-Roch theorem and Serre duality yields for compact Riemann surfaces $M_{\text {red }}$ of genus $g \geqq 2$, the case of main interest in the further text,

$$
\begin{aligned}
& \operatorname{dim} H^{1}\left(M_{\text {red }}, \mathcal{O}\left(K^{-1}\right)\right)=3 g-3, \\
& \operatorname{dim} H^{1}\left(M_{\text {red }}, \mathcal{O}\left(K^{-\frac{1}{2}}\right)\right)=2 g-2 .
\end{aligned}
$$

Since higher order deformations of the superconformal structure are either completely fixed by first order deformations or just linear combinations of them, the dimension of super moduli space is determined to be

$$
\operatorname{dim} \mathscr{M}_{g}=(3 g-3 \mid 2 g-2), \quad g \geqq 2 .
$$

4.2. Purely Odd Deformations. In order to get some information about holomorphic sections of line bundles over super Riemann surfaces, it is helpful to consider fibers of a differentiable family of compact super Riemann surfaces, which belongs to a fixed value $\left(t^{1}, \ldots, t^{m}\right)$ of the even moduli parameters. Let $M_{\zeta}:=\varpi^{-1}\left(t^{i}, \zeta^{j}\right)$ be the super Riemann surface corresponding to the point $\left(t^{i}, \zeta^{j}\right)$ of the parameter space - the reference to the fixed even parameters will be omitted in this section and $L_{\zeta}$ a holomorphic line bundle over $M_{\zeta}$. Then one finds the following

Theorem. On a compact super Riemann surface the dimension of the space of holomorphic sections of a holomorphic line bundle $\operatorname{dim} H^{0}\left(M_{\zeta}, \mathcal{O}\left(L_{\zeta}\right)\right)$ is independent of the odd moduli.

Proof. Denote by $M_{0}$ a split super Riemann surface, i.e. a fiber over some fixed point of the reduced parameter space. The transition functions between two open domains $U_{\alpha}, U_{\beta} \subset M_{0}$ can be written in the form

$$
\left(z_{\alpha}, \theta_{\alpha}\right)=\left\{f_{\alpha \beta, 0}^{z}\left(z_{\beta}\right), \theta_{\beta} f_{\alpha \beta, 0}^{\theta}\left(z_{\beta}\right)\right\} .
$$


A holomorphic line bundle $L_{0} \rightarrow M_{0}$ is described by a 1-cocycle with values in the nonvanishing holomorphic functions on $M_{0}$,

$$
\begin{aligned}
{\left[g_{\alpha \beta}\right] } & \in H^{1}\left(M_{0}, \mathcal{O}_{0}^{\times}\right), \\
g_{\alpha \beta}\left(z_{\beta}, \theta_{\beta} ; 0\right) & =g_{\alpha \beta, 0}\left(z_{\beta}\right) .
\end{aligned}
$$

Varying only the odd moduli $\left(\zeta^{1}, \ldots, \zeta^{n}\right)$, one obtains a super Riemann surface $M_{\zeta}$ with glueing functions (the argument $z_{\beta}$ will be omitted)

$$
\begin{aligned}
\left(z_{\alpha}, \theta_{\alpha}\right)= & \left\{f_{\alpha \beta, 0}^{z}+\zeta^{i} \theta_{\beta} f_{\alpha \beta, i}^{\theta}+\zeta^{i} \zeta^{j} f_{\alpha \beta, i j}^{z}+\cdots, \theta_{\beta} f_{\alpha \beta, 0}^{\theta}+\zeta^{i} f_{\alpha \beta, i}^{z}\right. \\
& \left.+\zeta^{i} \zeta^{j} \theta_{\beta} f_{\alpha \beta, i j}^{\theta}+\cdots\right\},
\end{aligned}
$$

and a deformed line bundle $L_{\zeta} \rightarrow M_{\zeta}$ with transition functions

$$
\begin{aligned}
{\left[g_{\alpha \beta}\right] } & \in H^{1}\left(M_{\zeta}, \mathcal{O}_{\zeta}^{\times}\right), \\
g_{\alpha \beta}\left(z_{\beta}, \theta_{\beta} ; \zeta\right) & =g_{\alpha \beta, 0}+\theta_{\beta} \zeta^{i} g_{\alpha \beta, i}+\zeta^{i} \zeta^{j} g_{\alpha \beta, i j}+\cdots .
\end{aligned}
$$

Now consider a global holomorphic section of $L_{\zeta}$, represented by a collection of holomorphic functions

$$
\left\{\sigma_{\alpha}\right\} \in H^{0}\left(M_{\zeta}, \mathcal{O}\left(L_{\zeta}\right)\right)
$$

which are glued on the intersection of two open domains $U_{\alpha} \cap U_{\beta} \subset M_{\zeta}$ by the prescription

$$
\sigma_{\alpha}\left(z_{\alpha}, \theta_{\alpha} ; \zeta\right)=g_{\alpha \beta}\left(z_{\beta}, \theta_{\beta} ; \zeta\right) \sigma_{\beta}\left(z_{\beta}, \theta_{\beta} ; \zeta\right)
$$

Using the notation

$$
\sigma_{\alpha}\left(z_{\alpha}, \theta_{\alpha} ; \zeta\right)=\sigma_{\alpha, 0}\left(z_{\alpha}, \theta_{\alpha}\right)+\zeta^{i} \sigma_{\alpha, i}\left(z_{\alpha}, \theta_{\alpha}\right)+\zeta^{i} \zeta^{j} \sigma_{\alpha, i j}\left(z_{\alpha}, \theta_{\alpha}\right)+\cdots,
$$

a power series expansion of Eq. (4.17) with respect to the odd parameters $\zeta^{j}$ results in consistency relations for each of the finitely many coefficient functions of the section $\left\{\sigma_{\alpha}\right\}$. Namely, one has

$$
\sigma_{\alpha, 0}\left(f_{\alpha \beta, 0}^{z}, \theta_{\beta} f_{\alpha, 0}^{\theta}\right)=g_{\alpha \beta, 0} \sigma_{\beta, 0}\left(z_{\beta}, \theta_{\beta}\right)
$$

in the lowest order. The first order terms yield

$$
\begin{array}{r}
\sigma_{\alpha, i}\left(f_{\alpha \beta, 0}^{z}, \theta_{\beta} f_{\alpha \beta, 0}^{\theta}\right)+\frac{\partial \sigma_{\alpha, 0}}{\partial z_{\alpha}}\left(f_{\alpha \beta, 0}^{z}, \theta_{\beta} f_{\alpha \beta, 0}^{\theta}\right) \theta_{\beta} f_{\alpha \beta, i}^{\theta}+\frac{\partial \sigma_{\alpha, 0}}{\partial \theta_{\alpha}}\left(f_{\alpha \beta, 0}^{z}, \theta_{\beta} f_{\alpha \beta, 0}^{\theta}\right) f_{\alpha \beta, i}^{z} \\
=g_{\alpha \beta, 0} \sigma_{\beta, i}\left(z_{\beta}, \theta_{\beta}\right)-\theta_{\beta} g_{\alpha \beta, i} \sigma_{\beta, 0}\left(z_{\beta}, \theta_{\beta}\right)
\end{array}
$$

and writing as a shorthand notation

$$
\sigma_{\alpha, \cdot}=\sigma_{\alpha, \cdot}\left(f_{\alpha \beta, 0}^{z}, \theta_{\beta} f_{\alpha \beta, 0}^{\theta}\right), \quad \sigma_{\beta, \cdot}=\sigma_{\beta, \cdot}\left(z_{\beta}, \theta_{\beta}\right),
$$

one finds in second order

$$
\begin{array}{r}
\sigma_{\alpha, i j}+\frac{\partial \sigma_{\alpha, i}}{\partial z_{\alpha}} \theta_{\beta} f_{\alpha \beta, j}^{\theta}+\frac{\partial \sigma_{\alpha, i}}{\partial \theta_{\alpha}} f_{\alpha \beta, j}^{z}+\frac{\partial \sigma_{\alpha, 0}}{\partial z_{\alpha}} f_{\alpha \beta, i j}^{z}+\frac{\partial \sigma_{\alpha, 0}}{\partial \theta_{\alpha}} \theta_{\beta} f_{\alpha \beta, i j}^{\theta} \\
=g_{\alpha \beta, 0} \sigma_{\beta, i j}+\theta_{\beta} g_{\alpha \beta, i}^{\theta} \sigma_{\beta, j}+g_{\alpha \beta, i j} \sigma_{\beta, 0} !
\end{array}
$$


As can be seen easily, comparing coefficients of the $k^{\text {th }}$ order lead to a relation of the form

$$
\begin{aligned}
& \sigma_{\alpha, i_{1} \ldots i_{k}}\left(f_{\alpha \beta, 0}^{z}, \theta_{\beta} f_{\alpha \beta, 0}^{\theta}\right)+\left\{\text { terms in } \sigma_{\alpha, i_{1} \ldots i_{l}}(l<k)\right\} \\
& \quad=g_{\alpha \beta, 0} \sigma_{\alpha, i_{1} \ldots i_{k}}\left(z_{\beta}, \theta_{\beta}\right)+\left\{\text { terms in } \sigma_{\alpha, i_{1} \ldots i_{l}}(l<k)\right\} .
\end{aligned}
$$

The next step is a discussion of the possible solutions of the consistency relations restricting the coefficient functions of the section $\left\{\sigma_{\alpha}\right\}$. At first notice that (4.19) characterizes $\left\{\sigma_{\alpha, 0}\right\}$ as a section of the bundle $L_{0}$ over the split super Riemann surface $M_{0}$. Secondly, if there exists a solution of Eq. (4.23), this solution is determined only up to addition of sections of the line bundle $L_{0} \rightarrow M_{0}$. Therefore the lowest coefficient function $\left\{\sigma_{\alpha, 0}\right\}$ of a section of $L_{\zeta} \rightarrow M_{\zeta}$ fixes all higher order functions up to addition of further elements of $H^{0}\left(M_{0}, \mathcal{O}\left(L_{0}\right)\right)$, so

$$
\operatorname{dim} H^{0}\left(M_{\zeta}, \mathcal{O}\left(L_{\zeta}\right)\right) \leqq \operatorname{dim} H^{0}\left(M_{0}, \mathcal{O}\left(L_{0}\right)\right) .
$$

If solutions of (4.23) only exist for $k<n$, an ansatz of the form

$$
\begin{gathered}
\sigma_{\alpha}\left(z_{\alpha}, \theta_{\alpha} ; \zeta\right)=\zeta^{i_{1}} \ldots \zeta^{i_{N-n+1}} \sigma_{\alpha, i_{1} \ldots i_{N-n+1}}\left(z_{\alpha}, \theta_{\alpha}\right)+\cdots+\zeta^{i_{1}} \ldots \zeta^{i_{N}} \sigma_{\alpha, i_{1}} \ldots i_{N}\left(z_{\alpha}, \theta_{\alpha}\right), \\
N:=\operatorname{odd} \operatorname{dim} \mathscr{M}_{g}=2 g-2 \quad(g \geqq 2)
\end{gathered}
$$

gives a global section of $L_{\zeta} \rightarrow M_{\zeta}$. In this case $\left\{\sigma_{\alpha, i_{1} \ldots i_{N-n+1}}\right\}$ obeys relations analogous to (4.19), and the $n^{\text {th }}$ order equation, which was the reason for the obstruction, does not appear anymore.

Finally one observes that the glueing functions $f_{\alpha \beta}\left(z_{\beta}, \theta_{\beta} ; \zeta\right)$ and the bundle transition functions $g_{\alpha \beta}\left(z_{\beta}, \theta_{\beta} ; \zeta\right)$ are per definitionem always holomorphic in the relative fiber coordinates $z, \theta$. Furthermore a section $\left\{\sigma_{\alpha}\right\} \in H^{0}\left(M_{\zeta}, \mathcal{O}\left(L_{\zeta}\right)\right)$ depends differentiably on the odd parameters $\zeta^{j}$, but since the property of being holomorphic in the relative fiber coordinates $z, \theta$ is a local property, one only has to ensure that each coefficient function in the power series expansion of $\left\{\sigma_{\alpha}\right\}$ with respect to $\zeta^{j}$ is holomorphic.

To summarize, an arbitrary holomorphic section of $L_{0} \rightarrow M_{0}$ always allows the construction of a holomorphic section of $L_{\zeta} \rightarrow M_{\zeta}$, which together with relation (4.24) leads to

$$
\operatorname{dim} H^{0}\left(M_{\zeta}, \mathcal{O}\left(L_{\zeta}\right)\right)=\operatorname{dim} H^{0}\left(M_{0}, \mathcal{O}\left(L_{0}\right)\right)
$$

and finishes the proof.

A direct consequence of Eq. (4.26) is the following

Theorem. (Riemann-Roch). Let $M$ be a compact super Riemann surface of genus $g$ and $L$ a holomorphic line bundle over $M$, then

$$
\operatorname{dim} H^{0}(M, \mathcal{O}(L))-\operatorname{dim} H^{1}(M, \mathcal{O}(L))-(1 \mid 1) \cdot c(L)=(1-g \mid 0) .
$$

Proof. Because of (4.26) the super Riemann surface $M$ can be considered to be split. In this case by (3.22) and (4.1) the cohomology groups $H^{\circ}(M, \mathcal{O}(L))$ are direct sums of some cohomology groups on the reduced Riemann surface $M_{\text {red }}$,

$$
H^{\cdot}(M, \mathcal{O}(L))=H^{\cdot}\left(M_{\text {red }} \mathcal{O}\left(L_{\text {red }}\right)\right) \oplus \Pi H^{\cdot}\left(M_{\text {red }}, \mathcal{O}\left(L_{\text {red }} \otimes \omega_{\text {red }}\right)\right),
$$


where the so-called parity change functor $\Pi[28]$ is used to achieve the correct parity of the second term. Therefore application of the ordinary Riemann-Roch theorem gives

$\operatorname{dim} H^{0}(M, \mathcal{O}(L))-\operatorname{dim} H^{1}(M, \mathcal{O}(L))-(1 \mid 1) \cdot c(L)=\left(1-g \mid 1-g+c\left(\omega_{\text {red }}\right)\right)$,

yielding the desired result with the help of (3.24).

\section{Hyperbolic Geometry on $\mathscr{H}^{1 \mid 1}$}

A more explicit way to represent compact super Riemann surfaces of genus $g \geqq 2$ is the tessellation of the super Poincare upper half-plane by Fuchsian groups. As a preparation I will first consider superconformal automorphisms of the generalized Riemann sphere. Automorphic forms will be introduced as the analogs of sections of tensor powers of the canonical bundle over a super Riemann surface. The last part of this chapter deals with the super Laplace operator $\square_{p}$ acting on automorphic forms, especially with the number of linear independent zero modes of $\square_{p}$, which is of interest in the calculation of the fermionic string partition function.

5.1. Uniformization. One of the classical theorems of complex analysis states that every simply connected Riemann surface is conformally equivalent to $\hat{\mathbb{C}}=\mathbb{C} \cup\{\infty\}, \mathbb{C}$ or $\mathscr{H}$ (Riemann, Poincaré, Koebe). As was shown by Crane and Rabin [7], unique generalizations $\hat{\mathbb{C}}^{1 \mid 1}, \mathbb{C}^{1 \mid 1}, \mathscr{H}^{1 \mid 1}$ of these Riemann surfaces exist. The reason is that the superconformal structure on $\hat{\mathbb{C}}^{1 \mid 1}, \mathbb{C}^{1 \mid 1}$ or $\mathscr{H}^{1 \mid 1}$ can always be described by coordinates, whose transition functions have the form (4.1), so like every split super Riemann surface $\hat{\mathbb{C}}^{1 \mid 1}, \mathbb{C}^{1 \mid 1}$ and $\mathscr{H}^{1 \mid 1}$ are completely determined by their reduced spaces.

The super Riemann sphere $\hat{\mathbb{C}}^{1 / 1}$ can be covered by two open domains, glued by

$$
\left(z^{\prime}, \theta^{\prime}\right)=\left(-\frac{1}{z}, \frac{\theta}{z}\right) .
$$

But to determine the superconformal automorphisms of $\hat{\mathbb{C}}^{1 \mid 1}$ it is more convenient to use homogeneous coordinates and to think of $\hat{\mathbb{C}}^{1 \mid 1}$ as a projective space,

$$
\begin{gathered}
\left(z_{1}, z_{2}, \eta\right) \in\left(\mathbb{C}^{2 \mid 1}\right)^{\times}:=\left\{x \in \mathbb{C}^{2 \mid 1} \mid x \bmod \mathscr{N} \neq 0\right\} \\
\left(z_{1}, z_{2}, \eta\right) \sim\left(z_{1}^{\prime}, z_{2}^{\prime}, \eta^{\prime}\right): \Leftrightarrow \exists \lambda \in\left(\mathbb{C}^{1 \mid 0}\right)^{\times}:\left(z_{1}, z_{2}, \eta\right)=\left(\lambda z_{1}, \lambda z_{2}, \lambda \eta\right) \\
\mathbb{P}^{1 \mid 1}(\mathbb{C}):=\left(\mathbb{C}^{2 \mid 1}\right)^{\times} / \sim
\end{gathered}
$$

The notation $\lambda \in\left(\mathbb{C}^{1 / 0}\right)^{\times}$means that $\lambda$ is allowed to contain odd parameters, since implicitly always families of supermanifolds will be considered. Expressing the local coordinates on the two charts of $\hat{\mathbb{C}}^{1 \mid 1}$ by the homogeneous coordinates of $\mathbb{P}^{1 \mid 1}(\mathbb{C})$,

$$
(z, \theta)=\left(\frac{z_{1}}{z_{2}}, \frac{\eta}{z_{2}}\right), \quad\left(z^{\prime}, \theta^{\prime}\right)=\left(-\frac{z_{2}}{z_{1}}, \frac{\eta}{z_{1}}\right),
$$


Aut $\widehat{\mathbb{C}}^{1 \mid 1}$ ' consists of invertible linear maps on $\mathbb{P}^{1 \mid 1}(\mathbb{C})$, being superconformal in the local coordinates ${ }^{2}(5.3)$,

$$
\text { Aut } \widehat{\mathbb{C}}^{1 \mid 1} \subset \mathrm{GL}(2 \mid 1, \mathbb{C})
$$

Writing

$$
x^{\prime}=\gamma x, \quad x, x^{\prime} \in \mathbb{P}^{1 \mid 1}(\mathbb{C}), \quad \gamma=\left(\begin{array}{lll}
a & b & \varepsilon \\
c & d & \delta \\
\alpha & \beta & e
\end{array}\right) \in \mathrm{GL}(2 \mid 1, \mathbb{C})
$$

yields

$$
z^{\prime}=\frac{a z+b+\varepsilon \theta}{c z+d+\delta \theta}, \quad \theta^{\prime}=\frac{\alpha z+\beta+e \theta}{c z+d+\delta \theta},
$$

if $z, \theta$ and $z^{\prime}, \theta^{\prime}$ are the local coordinates of $x, x^{\prime} \in \mathbb{P}^{1 \mid 1}(\mathbb{C})$, respectively. Ensuring the map (5.6) to be superconformal, the condition (3.4) results in (defining $k:=a d-b c)$

$$
\begin{aligned}
& k+\beta \alpha+2 \delta \varepsilon=e^{2}, \\
& \alpha e+\varepsilon c-\delta a=0, \\
& \beta e+\varepsilon d-\delta b=0 .
\end{aligned}
$$

Eliminating $e, \delta$ and $\varepsilon$, a square root ambiguity appears, which can be removed by introducing a character $v_{\gamma}= \pm 1$,

$$
\begin{aligned}
& e= \pm \sqrt{k}\left(1+\frac{3}{2 k} \beta \alpha\right)=: v_{\gamma} \sqrt{k}\left(1+\frac{3}{2 k} \beta \alpha\right) \\
& \delta=v_{\gamma} \sqrt{k}(\alpha d-\beta c) \\
& \varepsilon=v_{\gamma} \sqrt{k}(\alpha b-\beta a) .
\end{aligned}
$$

Calculating the determinant of the transformation $\gamma$, one finds

$$
\operatorname{sdet} \gamma=v_{\gamma} \sqrt{k}\left\{1+\left(\frac{3}{2 k}-2\right) \alpha \beta\right\},
$$

but since $\gamma$ acts on homogeneous coordinates of $\mathbb{P}^{1 \mid 1}(\mathbb{C})$, without loss of generality the choice sdet $\gamma \stackrel{!}{=} v_{\gamma}= \pm 1$ can be made, leading to

$$
k=1+\alpha \beta .
$$

Inserting into (5.8) one obtains the general form of a superconformal automorphism on $\hat{\mathbb{C}}^{1 \mid 1}$,

$$
\gamma=\left(\begin{array}{lll}
a & b & v_{\gamma}(\alpha b-\beta a) \\
c & d & v_{\gamma}(\alpha d-\beta c) \\
\alpha & \beta & v_{\gamma}(1+\beta \alpha)
\end{array}\right), \quad a d-b c=1+\alpha \beta
$$

\footnotetext{
${ }^{2}$ Simplifying notation, I will write $\mathrm{GL}(2 \mid 1, \mathbb{C})$ even if the entries of a matrix are allowed to have odd parity. Odd variables will be denoted by Greek letters as usual.
} 
In the absence of any odd parameters, $\gamma$ reduces to an ordinary Möbius transformation (with $a d-b c=1$ ) accompanied by a character $v_{\gamma}= \pm 1$.

To characterize Aut $\hat{\mathbb{C}}^{1 \mid 1}$ as a subgroup of $\operatorname{GL}(2 \mid 1, \mathbb{C})$, notice that every $\gamma \in$ Aut $\hat{\mathbb{C}}^{1 / 1}$ leaves invariant the canonical alternating bilinear form on $\mathbb{C}^{2 \mid 1}$,

$$
I:=\left(\begin{array}{rrr}
0 & 1 & 0 \\
-1 & 0 & 0 \\
0 & 0 & 1
\end{array}\right),
$$

i.e. for all $\gamma \in \operatorname{GL}(2 \mid 1, \mathbb{C})$ fulfilling $\operatorname{sdet} \gamma= \pm 1$ the relations (5.7) are equivalent to

$$
\gamma^{\text {st }} I \gamma=I
$$

Matrices $\gamma$ having this property make up the superanalogue $\operatorname{OSp}(2 \mid 1, \mathbb{C})$ of the symplectic group $[1,28,33]$, and since $\gamma$ and $-\gamma$ describe the same element in Aut $\hat{\mathbb{C}}^{1 \mid 1}$, finally

$$
\text { Aut } \widehat{\mathbb{C}}^{1 \mid 1}=\frac{\operatorname{OSp}(2 \mid 1, \mathbb{C})}{\{ \pm \mathbb{1}\}}
$$

Acting on local coordinates, a superconformal automorphism of the super Riemann sphere takes the form

$$
\begin{aligned}
& z^{\prime}=\frac{a z+b}{c z+d}+v_{\gamma} \theta \frac{\alpha z+\beta}{(c z+d)^{2}}, \\
& \theta^{\prime}=\frac{\alpha z+\beta}{c z+d}+\frac{v_{\gamma} \theta}{c z+d} .
\end{aligned}
$$

The transformation rule for the $D$-operator (3.6) can be seen to be

$$
D^{\prime}=F_{\gamma} D, \quad F_{\gamma}:=\left(D \theta^{\prime}\right)^{-1}=v_{\gamma}(c z+d+\delta \theta) \text {. }
$$

To find the superconformal automorphisms of the super Poincaré upper halfplane $\mathscr{H}^{1 \mid 1}:=\left\{(z, \theta) \in \mathbb{C}^{1 \mid 1} \mid \mathfrak{F}(z) \bmod \mathscr{N}>0\right\}$, one considers the two conditions $\gamma(z, \theta) \in$ $\mathscr{H}^{1 \mid 1}, \forall(z, \theta) \in \mathscr{H}^{1 \mid 1}$ and $\gamma^{2} \in$ Aut $\mathscr{H}^{1 \mid 1}$, which can be fulfilled by choosing all coefficients in the map (5.15) to be real

$$
\text { Aut } \mathscr{H}^{1 \mid 1}=\frac{\operatorname{OSp}(2 \mid 1, \mathbb{R})}{\{ \pm \mathbb{1}\}}
$$

In complete analogy to the classical case, every compact super Riemann surface $M$ of genus $g \geqq 2$ can be represented $[3,23]$ as a quotient space $\mathscr{H}^{1 \mid 1} / \Gamma$, where $\Gamma$ denotes a discrete subgroup of Aut $\mathscr{H}^{1 \mid 1}$ having no fixed points. $\Gamma$ is isomorphic to the first homotopy group of the reduced Riemann surface $\Gamma \cong \pi_{1}\left(M_{\text {red }}\right)$. A transformation (5.11) is said to act discretely and without fixed points, if the corresponding transformation $\gamma \bmod \mathscr{N} \in \operatorname{SL}(2, \mathbb{R})$ has this property. Therefore $\Gamma-\{\mathbb{1}\}$ contains only hyperbolic elements, i.e. $\gamma \bmod \mathscr{N}, \forall \gamma \in \Gamma-\{\mathbb{1}\}$ is hyperbolic in the usual sense $(\mid a+d) \bmod \mathscr{N} \mid>2)$.

Choosing for some element of Aut $\mathscr{H}^{1 \mid 1}$ a representative with $\operatorname{str} \gamma \bmod \mathscr{N}>0$, it is always possible [1] to achieve the form

$$
\gamma \sim\left(\begin{array}{ccc}
e^{\frac{l_{y}}{2}} & 0 & 0 \\
0 & e^{-\frac{l_{\gamma}}{2}} & 0 \\
0 & 0 & v_{\gamma}
\end{array}\right)
$$


by conjugation in Aut $\mathscr{H}^{1 \mid 1}$. Since the supertrace is invariant under conjugation, comparing with (5.11) yields

$$
2 \cosh \frac{l_{\gamma}}{2}=a+d+v_{\gamma} \alpha \beta
$$

$l_{\gamma}$ can be interpreted as a generalized length of the shortest geodesic in the homotopy class corresponding to $\gamma$.

5.2. Automorphic Forms. Let $M=\mathscr{H}^{1 \mid 1} / \Gamma, \Gamma \subset$ Aut $\mathscr{H}^{1 \mid 1}$ be a super Riemann surface of genus $g$. Differentiable sections of powers of the canonical bundle $\omega$ (in the sense of tensor products) can be represented on $\mathscr{H}^{1 \mid 1}$ by automorphic forms of weight $(p, q)$,

$$
\begin{aligned}
\mathscr{E}(p, q) & :=H^{0}\left(M, \mathscr{E}\left(\omega^{p} \otimes \bar{\omega}^{q}\right)\right) \\
& =\left\{f: \mathscr{H}^{1 \mid 1} \rightarrow \mathbb{C}^{1 \mid 1} \mid f(\gamma Z)=F_{\gamma}^{p} \bar{F}_{\gamma}^{q} f(Z), \forall \gamma \in \Gamma\right\},
\end{aligned}
$$

where the shorthand notation $Z:=(z, \theta)$ for the coordinates on the super Poincaré upper halfplane is used. Defining

$$
Y:=y+\frac{i}{2} \theta \bar{\theta}=\frac{z-\bar{z}-\theta \bar{\theta}}{2 i} \in \mathscr{E}(-1,-1),
$$

it is possible to construct an $\operatorname{OSp}(2 \mid 1, \mathbb{R})$-invariant volume form on $\mathscr{H}^{1 \mid 1}$,

$$
d V:=\frac{D^{*}(d Z) \otimes D^{*}(d \bar{Z})}{Y} .
$$

In contrast to the classical case, the scalar product on $\mathscr{E}(p, q)$,

$$
\langle f, g\rangle:=\int_{M} d V Y^{p+q} \overline{f g}, \quad \forall f, g \in \mathscr{E}(p, q)
$$

is not positive definite, $\langle f, f\rangle$ can even be complex-valued.

The operator $\bar{D}=\partial_{\bar{\theta}}+\bar{\theta} \partial_{\bar{z}}$ transforms under the action of $\gamma \in \Gamma, Z^{\prime}=\gamma Z$ according to

$$
\bar{D}^{\prime}=\bar{F}_{\gamma} \bar{D}
$$

(compare (5.16)), therefore $\bar{D}$ maps an automorphic form of weight $(p, 0)$ to $\mathscr{E}(p, 1)$. Writing $\bar{D}_{p} \equiv \bar{D}$ to indicate more clearly the weight of the automorphic forms on which $\bar{D}$ acts, the scalar product (5.23) allows to define the adjoint operator $\bar{D}_{p}^{+}$,

$$
\begin{aligned}
\bar{D}_{p}: \mathscr{E}(p, 0) \leftrightarrow \mathscr{E}(p, 1): \bar{D}_{p}^{+}, & \left\langle\bar{D}_{p}^{+} f, g\right\rangle:=(-1)^{\tilde{f}}\left\langle f, \bar{D}_{p} g\right\rangle \\
& \Rightarrow \bar{D}_{p}^{+}=-\left\{Y D+\frac{i}{2} p(\bar{\theta}-\theta)\right\},
\end{aligned}
$$

$\tilde{f}$ denoting the parity of the values (!) of $f$. Using $\bar{D}_{p}^{+}$, the Laplace operator on the super Riemann surface $M$ reads

$$
\square_{p}: \mathscr{E}(p, 0) \rightarrow \mathscr{E}(p, 0), \quad \square_{p}:=-2 \bar{D}_{p}^{+} \bar{D}_{p} \Rightarrow \square_{p}=2 Y D \bar{D}+i p(\bar{\theta}-\theta) \bar{D}
$$

Comparing with the ordinary Laplacian

$$
\Delta_{p}: \mathscr{E}_{\text {red }}(p, 0) \rightarrow \mathscr{E}_{\text {red }}(p, 0), \quad \Delta_{p}:=4 \bar{\partial}_{p}^{+} \bar{\partial}_{p}=-4 y^{2} \partial_{z} \partial_{\bar{z}}+2 i p y \partial_{\bar{z}}
$$


the relation

$$
\square_{p}^{2} \bmod \mathscr{N}=\Delta_{p}
$$

holds.

A consequence of the scalar product (5.23) failing to be positive definite is that at first sight there is no such simple connection between $\operatorname{dim} \operatorname{ker} \square_{p}$ and $\operatorname{dim} \operatorname{ker} \bar{D}_{p}$ as the corresponding one in the classical case

$$
\operatorname{ker} \Delta_{p}=\operatorname{ker} \bar{\partial}_{p}=H^{0}\left(M, \mathcal{O}\left(K^{p / 2}\right)\right),
$$

i.e. classically the harmonic $p$-forms are just the holomorphic forms of weight $p$. In order to determine dim ker $\square_{p}$ one proceeds as follows: For a Fuchsian group $\Gamma \subset$ Aut $\mathscr{H}^{1 \mid 1}$ containing no odd parameters - in this case the super Riemann surface $M=\mathscr{H}^{1 / 1} / \Gamma$ is split - automorphic forms can be invariantly decomposed as

$$
f(Z, \bar{Z})=f_{0}(z, \bar{z})+\theta f_{1}(z, \bar{z})+\bar{\theta} f_{2}(z, \bar{z})+\bar{\theta} \theta f_{3}(z, \bar{z})
$$

where each $f_{i}(z, \bar{z})$ is a well defined classical automorphic form. Evaluating $\square_{p} f \stackrel{!}{=} 0$ leads to

$$
\begin{gathered}
\Delta_{p} f_{0}=0, \quad f_{3}=0, \\
(z-\bar{z}) \partial_{\bar{z}} f_{1}=-p f_{2}, \quad\left\{(z-\bar{z}) \partial_{z}+p\right\} f_{2}=0 .
\end{gathered}
$$

The last two relations can be rearranged to give

$$
\begin{gathered}
p=0: \quad \partial_{\bar{z}} f_{1}=0, \quad \partial_{z} f_{2}=0, \\
p \neq 0: \quad \Delta_{p+1} f_{1}=0, \quad f_{2}=-\frac{1}{p}(z-\bar{z}) \partial_{\bar{z}} f_{1},
\end{gathered}
$$

but using (5.29) one finds $f_{2}=0$ for $p \neq 0$. So on split super Riemann surfaces the number of linear independent harmonic $p$-forms is given by

$$
\begin{aligned}
& \operatorname{dim} \operatorname{ker} \square_{0}=\left(\operatorname{dim} \operatorname{ker} \Delta_{0} \mid 2 \operatorname{dim} \operatorname{ker} \bar{\partial}_{1}\right), \\
& \operatorname{dim} \operatorname{ker} \square_{p}=\left(\operatorname{dim} \operatorname{ker} \Delta_{p} \mid \operatorname{dim} \operatorname{ker} \Delta_{p+1}\right), \quad \forall p \neq 0 .
\end{aligned}
$$

To calculate $\operatorname{dim}$ ker $\square_{p}$ in the case of $M$ being a non-split super Riemann surface, the same formalism as in Sect. 4.2 applies. Namely, deforming a split super Riemann surface in the "odd direction," and using the fact that the property of a section $\sigma \in H^{0}\left(M, \mathscr{E}\left(\omega^{p}\right)\right)$ to be harmonic is a local one, the invariance of dim ker $\square_{p}$ can easily be seen. So the classical Riemann-Roch theorem yields for super Riemann surfaces of genus $g$

\begin{tabular}{|l|c|}
\hline weight & $\operatorname{dim}$ ker $\square_{p}$ \\
\hline$p \leqq-2$ & $(0 \mid 0)$ \\
$p=-1$ & $(0 \mid 1)$ \\
$p=0$ & $(1 \mid 2 q)$ \\
$p=1$ & $(q \mid g)$ \\
$p=2$ & $(g \mid 2 g-2)$ \\
$p \geqq 3$ & $((p-1)(g-1) \mid p(g-1))$ \\
\hline
\end{tabular}


The number of linear independent Dirac zero modes or harmonic spinors $q:=\operatorname{dim} \operatorname{ker} \bar{\partial}_{1}$ is not topologically invariant. Therefore it is impossible to calculate $q$ using the Riemann-Roch theorem. But on a Riemann surface with fixed spin structure, $q \bmod 2$ is invariant under deformation of the complex structure, i.e. independent of the moduli [2]. In [22] it is shown that generally $q$ is a discontinuous function of the moduli, restricted by the relation

$$
q \leqq \frac{1}{2}(g+1)
$$

\section{Application to the Fermionic String}

The investigation of super Riemann surfaces was originally motivated by the study of fermionic string theory $[10,11,20,24]$. In this section I will point out the connection between worldsheet supersymmetry and the introduction of anticommuting coordinates, as well as the necessity of considering families of super Riemann surfaces in order to describe a ghost-free fermionic theory. Reducing Polyakov's functional integral to a finite dimensional integral over super moduli space, the string integrand contains some determinants of the super Laplace operator, which can be expressed by Selberg's super zeta functions. The explicit structure of these relation depends on $\operatorname{dim} \operatorname{ker} \square_{p}$, calculated in the preceding section.

\subsection{Supersymmetry. Using Polyakov's bosonic string action}

$$
S\left[h_{m n}, X^{\mu}\right]=\int_{M} d^{2} \sigma \sqrt{h} h^{m n} \partial_{m} X^{\mu} \partial_{n} X^{\mu},
$$

the $g$-loop contribution to the partition function of the closed string can be written as a functional integral

$$
Z_{g}=\int \mathscr{D} h_{m n} \mathscr{D} X^{\mu} e^{-S\left[h_{m n}, X^{\mu}\right]}
$$

over all metrics $h_{m n}$ on the worldsheet $M$ and all embeddings $X: M \rightarrow \mathbb{R}^{d}$ of $M$ into $d$-dimensional euclidean spacetime. The Polyakov action is invariant under a change of the worldsheet coordinates and Weyl rescalings of the metric $h_{m n}$

$$
h_{m n}\left(\sigma^{k}\right) \rightarrow \Lambda\left(\sigma^{k}\right) h_{m n}\left(\sigma^{k}\right) .
$$

A detailed treatment shows a Weyl anomaly, which exactly cancels by choosing the dimension of spacetime to be $d=26$ [31]. So in the critical dimension the contribution of each surface $M$ to the partition function only depends on the conformal structure, leading to a reduction of (6.2) to a finite dimensional integral over the moduli space $\mathscr{M}_{g}^{R F}$ parametrizing all Riemann surfaces of genus $g$. Unfortunately the Fock space of the quantum theory described by the action (6.1) contains state vectors of negative norm (ghosts). Due to this fact a consistent probability interpretation seems to be impossible. A possible way out is a restriction of the Fock space by imposing the Gupta-Bleuler condition

$$
\left\langle b\left|T_{m n}\right| b\right\rangle=0
$$


i.e. for all physical states $|b\rangle$ the expectation value of the energy-momentum tensor

$$
T_{m n}=\frac{1}{\sqrt{h}} \frac{\delta S}{\delta h^{m n}}
$$

has to vanish.

In order to describe fermions too, one introduces some anticommuting sections $\psi^{\mu}$ of spinor bundles over $M$ besides the "bosonic fields" $X^{\mu}$ and extends the Polyakov action (6.1) to

$$
S_{F}[h, X, \psi]=\int_{M} d^{2} z \sqrt{h}\left(h^{z \bar{z}} \partial_{z} X^{\mu} \partial_{\bar{z}} X^{\mu}-\psi_{+}^{\mu} \partial_{\bar{z}} \psi_{+}^{\mu}-\psi_{-}^{\mu} \partial_{z} \psi_{-}^{\mu}\right) .
$$

In addition to reparametrization- and Weyl-invariance, the new action also is invariant under global worldsheet supersymmetry transformations

$$
\delta X^{\mu}=\zeta^{+} \psi_{+}^{\mu}+\zeta^{-} \psi_{-}^{\mu}, \quad \delta \psi_{+}^{\mu}=-\zeta^{+} \partial_{z} X^{\mu},
$$

where $\zeta \pm$ denotes a spinor, constant with respect to the worldsheet $M$. In the fermionic case, it has been found $[6,29,32]$, that a cancellation of the Weyl anomaly can be achieved by choosing the dimension of spacetime to be $d=10$. To eliminate ghost states in the quantized theory, one demands

$$
\left\langle f\left|T_{m n}\right| f\right\rangle=0,\left\langle f\left|J_{m n}\right| f\right\rangle=0,
$$

the second Gupta-Bleuler condition arising from an additional conserved current $J_{m n}$ corresponding to the worldsheet supersymmetry.

Calculating the commutator of two global supersymmetry transformations (6.7) results in (considering only the simpler case $\zeta^{-}=0$ )

$$
\begin{aligned}
& {\left[\delta_{S 1}, \delta_{S 2}\right] X^{\mu}=2 \zeta_{1}^{+} \zeta_{2}^{+} \partial_{z} X^{\mu},} \\
& {\left[\delta_{S 1}, \delta_{S 2}\right] \psi_{+}^{\mu}=2 \zeta_{1}^{+} \zeta_{2}^{+} \partial_{z} \psi_{+}^{\mu} .}
\end{aligned}
$$

Since the partial derivative $\partial_{z}$ can be considered as the generator of spatial translations

$$
\delta_{T} f(z):=f(z+e)-f(z)=e \partial_{z} f(z)+O\left(e^{2}\right),
$$

and spatial translations commute among themselves as well as with global supersymmetry transformations, the supersymmetry algebra reads

$$
\left[\delta_{T 1}, \delta_{T 2}\right]=0=\left[\delta_{S}, \delta_{T}\right], \quad\left[\delta_{S 1}, \delta_{S 2}\right]=2 \delta_{T} .
$$

Searching for a differential operator generating the supersymmetry transformations (6.7) fails on an ordinary Riemann surface, because $\delta_{S}$ mixes $X^{\mu}$ and $\psi^{\mu}$. But introducing an anticommuting complex coordinate $\theta$ besides $z$ on the worldsheet, and putting bosonic and fermionic fields together to build one superfield

$$
\Phi^{\mu}(z, \bar{z}, \theta)=X^{\mu}(z, \bar{z})+\theta \psi^{\mu}(z, \bar{z}),
$$

the transformations (6.7) can be written as

$$
\delta_{S} \Phi^{\mu}=\zeta^{+} D \Phi^{\mu}, \quad D=\partial_{\theta}+\theta \partial_{z} .
$$


Especially, acting on the coordinates $z, \theta$ one sees that supersymmetry transformations are generalized "translations" in superspace

$$
\begin{gathered}
\delta_{S} z=\zeta^{+} \theta, \quad \delta_{S} \theta=\zeta_{+} \\
\Rightarrow \delta_{S} \Phi^{\mu}=\Phi\left(z+\zeta^{+} \theta, \theta+\zeta^{+}\right)-\Phi^{\mu}(z, \theta) .
\end{gathered}
$$

The Gupta-Bleuler conditions (6.8) so far imposed "by hand" can be built in the theory by demanding the fermionic string action to be locally supersymmetric. This can only be done by introducing a superpartner of the worldsheet metric, the gravitino field $\chi$, which is an anticommuting section of the bundle $\bar{K} \otimes K^{-\frac{1}{2}}$. Now writing

$$
S[E, \Phi]=\int_{M} d(\mathrm{vol}) \operatorname{sdet} E_{M}{ }^{A} D \Phi^{\mu} \bar{D} \Phi^{\mu},
$$

where the superdeterminant of the frame field $E_{M}{ }^{A}$ is a function of the worldsheet metric and the gravitino field, describing the different geometries on the worldsheet, one has an action which is locally supersymmetric, i.e. invariant under a generalized version of the transformations (6.7) with $\zeta^{ \pm}$not necessarily constant [11]. Furthermore, the relations

$$
T:=\frac{1}{\sqrt{h}} \frac{\delta S}{\delta h}=0, \quad J:=\frac{1}{\sqrt{h}} \frac{\delta S}{\delta \chi}=0
$$

can be deduced from the action by using all invariances of $S[E, \Phi]$. So the corresponding string theory is free of ghosts from the beginning.

Investigating all invariances of the action (6.15), one observes, that $S[E, \Phi]$ only depends on the superconformal structure on $M$, so in complete analogy to the bosonic case the functional integral expressing the $g$-loop contribution to the fermionic partition function can be reduced to a finite dimensional integral over the moduli space $\mathscr{M}_{g}$ parametrizing all super Riemann surfaces of genus $g[4,9,21]$

$$
Z_{g}=\int_{\mathscr{M}_{g}} d(\operatorname{sWP})\left[\operatorname{sdet}^{\prime}\left(-\square_{0}^{2}\right)\right]^{-\frac{5}{2}}\left[\operatorname{sdet}^{\prime}\left(-\square-_{-2}^{2}\right)\right]^{\frac{1}{2}},
$$

$d$ (sWP) denoting the integration measure in $\mathscr{M}_{g}$, the Weil-Petersson measure, and the primes indicating suppression of zero modes.

It should be noted that the fermionic string has to be well distinguished from the Green-Schwarz superstring. Since the spectrum of the fermionic string - also denoted as the Ramond-Neveu-Schwarz string [20] - contains a tachyon, a truncation by means of the GSO-projection is necessary to obtain a consistent theory. This somewhat unnatural procedure can be avoided by using the Green-Schwarz superstring, which in addition to the worldsheet supersymmetry possesses a spacetime supersymmetry from the very beginning. Using the light-cone gauge quantization, it has been found that the spectra of the Green-Schwarz superstring and the Ramond-Neveu-Schwarz string with additionally imposed GSO-projection coincide [20].

6.2. Calculating the Partition Function. In the case of genus $g \geqq 2$ the determinants $\operatorname{sdet}\left(-\square_{p}{ }^{2}\right)$ can be expressed via Selberg's super zeta functions [21]

$$
Z_{r}(s):=\prod_{\{\gamma \in \Gamma\}_{p}} \prod_{k=0}^{\infty}\left[1-v_{\gamma}^{r} e^{-(s+k) l_{\nu}}\right], \quad \mathfrak{R}(s)>1, \quad r=0,1
$$


(cf. (5.19)). Defining

$$
\begin{aligned}
\Delta n^{(p)}:= & \left(\# \text { bosonic zero modes of } \square_{p}\right) \\
& -\left(\# \text { fermionic zero modes of } \square_{p}\right),
\end{aligned}
$$

table (5.34) shows that

$$
\Delta n^{(-2)}=0, \quad \Delta n^{(0)}=1-2 q
$$

Using the relation

$$
\operatorname{sdet}\left(\varepsilon^{2}-\square_{0}^{2}\right)=\frac{Z_{0}(1+\varepsilon) Z_{0}(\varepsilon)}{\left[Z_{1}\left(\frac{1}{2}+\varepsilon\right)\right]^{2}} \varepsilon^{2-2 g},
$$

one finds, since ord ${ }_{s=\frac{1}{2}} Z_{1}(s)=-\Delta n^{(0)}, Z_{1}\left(\frac{1}{2}+\varepsilon\right) \simeq: a \cdot \varepsilon^{2 q-1}$ and $Z_{0}(s)$ has a zero of order $2 g-2$ at $s=0$,

$$
\operatorname{sdet}\left(\varepsilon^{2}-\square_{0}^{2}\right) \stackrel{\varepsilon}{\simeq} \frac{1}{(2 g-2) !} \frac{Z_{0}(1) Z_{0}^{(2 g-2)}(0)}{a^{2}} \varepsilon^{2-4 q} .
$$

A consequence of the functional relation of Selberg's super zeta functions

$$
\frac{Z_{1}\left(\frac{1}{2}-s\right) Z_{0}(s)}{Z_{1}\left(\frac{1}{2}+s\right) Z_{0}(1-s)}=\frac{Z_{1}(0)}{Z_{1}(1)}(\sin \pi s)^{2 g-2}
$$

is

$$
Z_{0}^{(2 g-2)}(0)=(-1)^{\Delta n^{(0)}} \pi^{2 g-2}(2 g-2) ! Z_{0}(1) \frac{Z_{1}(0)}{Z_{1}(1)}
$$

so suppression of the zero modes leads to

$$
\operatorname{sdet}^{\prime}\left(-\square{ }_{0}^{2}\right)=(-1)^{1-2 q}\left[\pi^{g-1} \frac{Z_{0}(1)}{a}\right]^{2} \frac{Z_{1}(0)}{Z_{1}(1)} .
$$

For $p=-2$ no zero modes appear, and

$$
\lim _{\varepsilon \rightarrow 0} \operatorname{sdet}\left(\varepsilon^{2}-\square_{-2^{2}}{ }^{2}\right)=4^{1-g} \frac{(4 g-4) !}{(6 g-6) !} \frac{Z_{0}(2) Z_{0}^{(6 g-6)}(-1)}{\left.Z_{1}\right) Z_{1}^{(4 g-4)}\left(-\frac{1}{2}\right)}
$$

results in

$$
\operatorname{sdet}\left(-\square_{-2}{ }^{2}\right)=\left[\left(\frac{\pi}{2}\right)^{g-1} \frac{Z_{0}(2)}{Z_{1}\left(\frac{3}{2}\right)}\right]^{2} \frac{Z_{1}(0)}{Z_{1}(1)}
$$

Separating

$$
a=\left\{\begin{array}{cc}
\frac{Z_{1}^{(2 q-1)}\left(\frac{1}{2}\right)}{(2 q-1) !}, & 2 q-1>0, \\
\lim _{\varepsilon \rightarrow 0} \varepsilon^{1-2 q} Z_{1}\left(\frac{1}{2}+\varepsilon\right), & 2 q-1<0,
\end{array}\right.
$$


and using the relation (5.35), the 2-loop contribution to the fermionic partition function can be written as

$$
\begin{aligned}
Z_{g=2}= & \left(\frac{1}{2 \pi^{4}}\right)^{g-1} \int_{\mathscr{M}_{g=2}} d(\mathrm{sWP})\left(\frac{Z_{1}(1)}{Z_{1}(0)}\right)^{2} \frac{Z_{0}(2)}{Z_{1}\left(\frac{3}{2}\right)\left[Z_{0}(1)\right]^{5}} \\
& \cdot\left\{\begin{array}{cr}
(-i)\left[\operatorname{res}_{s=\frac{1}{2}} Z_{1}(s)\right]^{5}, & \text { even spin structure on } M_{\text {red }}, \\
i\left[Z_{1}^{\prime}\left(\frac{1}{2}\right)\right]^{5}, & \text { odd spin structure on } M_{\text {red }} .
\end{array}\right.
\end{aligned}
$$

However, since the Selberg super zeta functions are defined in terms of the geometrical structure, i.e. the length spectrum of the underlying surface, the lack of information about the complex structure makes it impossible to carry out the GSO-projection explicitly.

\section{Summary}

In this paper super Riemann surfaces were discussed from two different points of view. The description via glueing open superdomains was found to be very convenient for deforming the superconformal structure in the sense of the Kodaira-Spencer approach [25]. Representing super Riemann surfaces as a fundamental domain in the super Poincare upper half-plane results, on the other hand, in quite explicit relations offering (in my opinion) the simplest way to consider properties of the super Laplacian.

The central result proved in this work, the invariance of $\operatorname{dim} H^{0}\left(M_{\zeta}, \mathcal{O}\left(L_{\zeta}\right)\right)$ under purely odd deformations of super Riemann surfaces, has led to two consequences. On the mathematical side, a generalized version of the Riemann-Roch theorem has been established for compact super Riemann surfaces, and on the physical side, by giving the explicit relations expressing determinants of super Laplace operators in terms of Selberg's super zeta functions, at least the 2-loop contribution to the fermionic string partition function has been stated.

Acknowledgement. I would like to thank Jens Bolte for many stimulating discussions, as well as Prof. Frank Steiner for helpful remarks during the final stage of this work.

\section{References}

1. Aoki, K.: Heat Kernels and Superdeterminants of Laplace Operators on Super Riemann Surfaces. Commun. Math. Phys. 117, 405-429 (1988)

2. Atiyah, M.F.: Riemann Surfaces and Spin Structures. Ann. Sci. École Norm. Sup. 4, 47-62 (1971)

3. Baranov, M.A., Frolov, I.V., Shvarts, A.S.: Geometry of Two-dimensional Superconformal Field Theories. Transl. Teor. Mat. Fiz. 70, 64-72 (1987)

4. Baranov, M.A., Manin, Yu.I., Frolov, I.V., Schwarz, A.S.: A Superanalog of the Selberg Trace Formula and Multiloop Contributions for Fermionic Strings. Commun. Math. Phys. 111, 373-392 (1987)

5. Batchelor, M.: The Structure of Supermanifolds. Trans. Am. Math. Soc. 253, 329-338 (1979)

6. Chaudhuri, S., Kawai, H., Tye, S.-H.H.: Path-integral Formulation of Closed Strings. Phys. Rev. D36, 1148-1168 (1987)

7. Crane, L., Rabin, J.M.: Super Riemann Surfaces: Uniformization and Teichmüller Theory. Commun. Math. Phys. 113, 601-623 (1988) 
8. DeWitt, B.: Supermanifolds. Cambridge: Cambridge Univ. Press 1984

9. D'Hoker, E., Phong, D.H.: Superholomorphic Anomalies and Supermoduli Space. Nucl. Phys. B292, 317-329 (1987)

10. D'Hoker, E., Phong, D.H.: The Geometry of String Perturbation Theory. Rev. Mod. Phys. 60, 917-1065 (1988)

11. D'Hoker, E., Phong, D.H.: Superstrings, Super Riemann Surfaces, and Supermoduli Space. In: Proceedings of the "String Theory" Conference, Rome, Italy, June 1988

12. Falqui, G., Reina, C.: Supermoduli and Superstrings. In: Proceedings of the C.I.M.E. Summer Course "Global Geometry and Mathematical Physics", Montecattini, Italy, July 1988

13. Falqui, G., Reina, C.: A Note on the Global Structure of Supermoduli Space. Commun. Math. Phys. 128, 247-261 (1990)

14. Farkas, H.M., Kra, I.: Riemann Surfaces. Berlin, Heidelberg, New York: Springer 1980

15. Friedan, D.: Notes on String Theory and Two Dimensional Conformal Field Theory. In: Workshop Santa Barbara 1985: Unified String Theories, Green, M., Gross, D. (eds.) pp. $162-213$

16. Giddings, S.B.: A Brief Introduction to Super Riemann Surface Theory. In: Trieste School 1988: "Superstrings", pp. 251-275

17. Giddings, S.B., Nelson, P.: Torsion Constraints and Super Riemann Surfaces. Phys. Rev. Lett. 59, 2619-2622 (1987)

18. Giddings, S.B., Nelson, P.: The Geometry of Super Riemann Surfaces. Commun. Math. Phys. 116, 607-634 (1988)

19. Giddings, S.B., Nelson, P.: Line Bundles on Super Riemann Surfaces. Commun. Math. Phys. 118, 289-302 (1988)

20. Green, M.B., Schwarz, J.H., Witten, E.: Superstring Theory. Volume I, II. Cambridge: Cambridge Univ. Press 1987

21. Grosche, C.: Selberg Supertrace Formula for Super Riemann Surfaces, Analytic Properties of Selberg Super Zeta-Functions and Multiloop Contributions for the Fermionic String. Commun. Math. Phys. 133, 433-485 (1990)

22. Hitchin, N.: Harmonic Spinors. Adv. Math. 14, 1-55 (1974)

23. Hodgkin, L.: A Direct Calculation of Super-Teichmüller Space. Lett. Math. Phys. 14, 47-53 (1987)

24. Kaku, M.: Introduction to Superstrings. Berlin, Heidelberg, New York: Springer 1988

25. Kodaira, K.: Complex Manifolds and Deformations of Complex Structure. Berlin, Heidelberg, New York: Springer 1986

26. LeBrun, C., Rothstein, M.: Moduli of Super Riemann Surfaces. Commun. Math. Phys. 117, 159-176 (1988)

27. Leites, D.A.: Introduction to the Theory of Supermanifolds. Russ. Math. Surveys 35, 1-64 (1980)

28. Manin, Yu.I.: Gauge Field Theory and Complex Geometry. Berlin, Heidelberg, New York: Springer 1988

29. Martinec, E.: Superspace Geometry of Fermionic Strings. Phys. Rev. D28, 2604-2613 (1983)

30. Nelson, P.: Introduction to Supermanifolds. Int. J. Mod. Phys. A3, 585-590 (1988)

31. Polyakov, A.M.: Quantum Geometry of Bosonic Strings. Phys. Lett. 103B, 207-210 (1981)

32. Polyakov, A.M.: Quantum Geometry of Fermionic Strings. Phys. Lett. 103B, 211-213 (1981)

33. Rabin, J.M., Topiwala, P.: Super Riemann Surfaces are Algebraic Curves. Preprint 88-0569 (UC, San Diego), June 1988

34. Rogers, A.: Graded Manifolds, Supermanifolds and Infinite-Dimensional Grassmann Algebras. Commun. Math. Phys. 105, 375-384 (1986)

35. Rosly, A.A., Schwarz, A.S., Voronov, A.A.: Geometry of Superconformal Manifolds. Commun. Math. Phys. 119, 129-152 (1988)

Communicated by S.-T. Yau 